suggests putting some of the principles and tools of global logistics to the use of migrants. In the database MANY, which she presented at the Architecture Biennale in Venice in 2018, the African Terminal featured prominently as one of her examples. The idea of starting to build supply chains based on migrational expertise seems to be promising. Imagine that migration would no longer be seen as a journey from beginning to end, as leaving one country behind to ideally become citizen of another, but as an endless entanglement of people and things in motion. Imagine that migrants would finally be acknowledged as experts in this regard. Imagine the multitude of alternative supply chains that people with migrational experience would be able to build. Imagine that they would not be constantly stopped but supported in this endeavor.

Imagine that we were able to relate differently. That we might act as if we were able to relate differently.

It might seem like a wide stretch from performing citizenship within the city to the negotiations and fights in rebuilding and reclaiming citizenship in the interconnectedness of transcontinental migration and sea trade. However, I would like to argue that in order to perform everybody's right to research in a city like Hamburg, and in most big cities today, this is exactly the tension we have to endure - in practice, theory, and research. Even if, for now, the results of this research will not foster global changes of structures but only microchanges, experiencing and understanding these changes has an effect on the collective imagination of what's possible and worth fighting for - as the citizen performers and citizen researchers we are today.

\title{
Tom Holert Verkomplizierung der Möglichkeiten: Gegenwartskunst, Epistemologie, Wissenspolitik
}

Wie lässt sich ein Meta-Diskurs über situierte Wissen und nichtwestliche Epistemologien im Kontext von Kunst und Kunsttheorie situieren? Vom Standpunkt der Standpunktsensibilität aus zu reflektieren, scheint im Licht einer solchen Frage gleichermaßen unausweichlich wie überaus widersprüchlich zu sein. Die Probleme, die sich in meinem Fall damit verbinden können, ja unweigerlich ergeben müssen, sind nicht über einen schlichten Sprechakt zu lösen, und eine ,Lösung ist auch nicht anzustreben, nicht nur weil sie aussichtslos erscheint. Im Unterschied, sagen wir, zu einer Forscherin mit einem Maori- oder Inuit-Hintergrund kann ich als Person, die sich, soweit dies die Dekonstruktionen des Autorenbegriffs zulassen, auch als Autor dieses Textes 
versteht, mich nicht oder zumindest nicht mit der gleichen Selbstverständlichkeit auf meine norddeutschen Wurzeln berufen, auf mein europäisches Weiß- und Mannsein. Und trotzdem sollte ich dies tun: weniger im Interesse einer Selbstvergewisserung und Authentifizierung meiner Sprecherposition in einer tribalen oder anderweitigen, mit Vorstellungen von Autochthonie durchsetzten Genealogie als im Hinblick auf meine unter anderem von der westlichen Moderne und westdeutschen Bildungsbegriffen geprägten, strukturierten und von mir entsprechend ein- und ausgeübten Weisen des Wissens. Dass eine solche selbstreflexive Geste in einem Fall wie dem meinen und in einem Setting wie dem des Forschungszusammenhangs, zu dem dieser Beitrag eingeladen wurde, aber automatisch irgendwie eitel, übertrieben, unangebracht und daher unselbstverständlich anmutet, lässt auf vieles schließen vor allem auf die Aporie, kritisch mit Privilegien umzugehen; auf Konventionen, die einen affirmativen Bezug auf eine Community im akademischen Kontext seltsam und unangebracht erscheinen lassen; womöglich auf die Ahnung eines Schuldzusammenhangs, der das Herausstellen von Herkunft im Fall eines weißen, männlichen, europäischen Akademikers zu einer Anmaßung werden lässt, der Anmaßung nämlich, sich wie jemand mit dem legitimen Anspruch, eine indigene, vom Aussterben bedrohte Bevölkerungsgruppe zu vertreten, aufzuführen, als unguter Aneignung einer eben nicht nur rituellen, sondern auch politischen Positionsbestimmung auf den Feldern des Wissens und der Kunst. ${ }^{1}$

Die Politiken des Wissens, die auf dem Feld der immer noch so bezeichneten Gegenwartskunst/contemporary art zu beobachten sind, gehören zu den genannten Problemen bzw. ergänzen diese. Denn es gibt nicht nur ein wie auch immer umstrittenes Wissen davon, was Kunst ist, was eine Künstler_in ist, was eine Kunstausstellung ist, sondern auch ein Wissen oder eine Vielzahl von Wissen, die sich in und durch Kunstwerke, das heißt durch als künstlerisch anerkannte Formen, Prozesse, Performanzen und Interaktionen, aber auch durch diskursive Rahmungen aller Art - vom Kurator_innen-Essay über das Interview oder den Vortrag bis zur Pressemitteilung - artikulieren.

Zum einen tragen diese Wissensformen zur fortwährenden Stabilisierung eines Glaubenssystems der Gegenwartskunst bei; zum anderen gehört es zum konzeptualistischen Selbstverständnis eines wichtigen Teils der machtvollen Formation der Gegenwartskunst, gegen hegemoniale und konventionalisierte, vor allem aber gegen als gewaltförmig auftretende, andere Wissensarten verdrängende und unterdrückende Wissensformen kritisch-dekonstruktiv vorzugehen - de-instituierend, ent-nennend, dis-identifizierend; womit aber auch, automatisch, der gesamte problematische Zusammenhang einer weiterhin

1 Scharfsinnig auf die genannten Probleme (inklusive einer kurzen kritischen Erwähnung der ursprünglichen, hier leicht revidierten Einleitung meines Vortrags während der „How to Relate“-Konferenz) gehen ein: Gramlich, Naomie / Haas, Annika: „Situiertes Schreiben mit Haraway, Cixous und grauen Quellen“, in: ZfM. Zeitschrift für Medienwissenschaft, Jg. 11, Nr. 1 (Zwanzig. Was uns angeht), 2019, S. 38-52. 
von westlich-modernen dichotomischen Vorstellungen geprägten und damit privilegierten, universalisierenden Rede von der Kunst (und der korrespondierenden Praxis) betroffen sein müsste.

Mit anderen Worten: Die Position der ,Kunst' im Sinne der globalen Gegenwartskunst einzunehmen, ist in der Konsequenz mit einer ähnlichen Widersprüchlichkeit und Problematik behaftet wie die Selbsteinführung, das diskursive Selfie als weißer, männlicher Westeuropäer. In diesem Sinne wären die Politiken des Wissens, wie sie auf dem Feld der Kunst präsent sind, auch entweder affirmative oder kritische - Operationen an den ontologischen und epistemologischen Prämissen einer immer weiter in Richtung Welt(markt-) kunst, global art, neuem Internationalismus tendierenden Entwicklung.

An den Umarmungen, den endorsements, den oft freundlich-gutmeinend daherkommenden, aber im Kern fragwürdigen bis feindlichen Übernahmen von nichtwestlichen, nicht- oder paramodernen Formen des Wissens und der Kunst, die auf diesem Feld stattfinden; an dem bemerkenswerten, aber auch oft genug problematischen Interesse an der Inklusion von zuvor Jahrzehnte, Jahrhunderte lang aus dem Kunstfeld ausgeschlossenen Praktiken und Traditionen des Wissens: an diesen Annäherungen, Begegnungen und Vereinnahmungen und dem Umgang mit (und dem Verhalten in) ihnen entscheidet sich, wie eine Politik des Wissens in den institutionellen, aber eben auch zunehmend gegen- oder para-institutionellen Kontexten der Kunst angelegt sein müsste. Und hieran knüpft des Weiteren die Herausforderung an, sich der Expansionslogik einer Kunstindustrie, die zu einem zentralen, gestaltend-einflussreichen Akteur der globalen Wissensökonomie geworden ist, erfolgreich zu entziehen oder deren Agenden zu durchkreuzen.

Die Frage nach Relation und Relationalität, nach Bezug und Bezüglichkeit, genauer: die Formulierung „How to relate“, die diesem Band vorangestellt ist, hat bei mir - déformation professionnelle eines mit der zeitgenössischen bildenden Kunst und ihren kuratorischen Konzepten seit Längerem befassten Autors - sogleich eine längst triviale Assoziation ausgelöst. Ich dachte an Esthétique relationnelle, den Titel von Nicolas Bourriauds Buch aus den späten 1990er Jahren, und die auf diesem Buch basierende Rede von „relationaler Kunst“.2 Sie wurde für die kunstkritischen Debatten der 2000er Jahre ähnlich bedeutsam wie die von Jacques Rancière - einem Gegner der Kunst, die von Bourriauds Begriff adressiert werden sollte - eingeführte „Aufteilung des Sinnlichen" (partage du sensible oder distribution of the sensible). ${ }^{3}$ Ich will hier nur daran erinnern, dass der Begriff „relationale Ästhetik“ auf einen Typ installativ-performativer Kunst angewendet wurde, die maßgeblich um soziale, vorderhand gastfreundliche Aktivität herum aufgebaut ist, etwa um Kochen im Ausstellungsraum oder die Herstellung eines fließenden Übergangs

2 Ich verwende hier die engl. Übersetzung: Bourriaud, Nicolas: Relational Aesthetics, Dijon 2002 [zuerst Dijon 1998].

3 Rancière, Jacques: Die Aufteilung des Sinnlichen. Die Politik der Kunst und ihre Paradoxien, Berlin 2006 [zuerst Paris 2000]. 
zwischen Orten der Kunstbetrachtung, des Diskurses, des Chillens und der Party. Rancière (und andere) kritisierten Bourriauds vermeintlich unpolitischen Ansatz, seine angeblich verharmlosend-verniedlichende Vorstellung von Kunst. ${ }^{4}$ Bourriaud hätte alle Errungenschaften einer modernen Ästhetik, die auf eine revolutionäre und streitbare Neuaufteilung, Entgrenzung und Unterwanderung traditioneller ästhetischer Hierarchien aus gewesen sei, aufgegeben - zugunsten einer nivellierenden, die Verhältnisse eines millenialen Stillstands allenfalls spiegelnden, diese aber nie herausfordernden und in Frage stellenden Mitmachkunst.

Im Frühjahr 2009 und somit etwa zehn Jahre nach dem Erscheinen von Esthétique relationnelle kuratierte Bourriaud, auch aufgrund seiner theoretischen Äußerungen längst zu einem der tonangebenden Kuratoren des globalen Kunstbetriebs aufgestiegen, in London die Ausstellung „Altermodern“. Verblüffend an dieser Ausstellung war nicht nur, dass die Kritik an seinem früheren Konzept offenbar kaum zu Bourriaud durchgedrungen war, sondern auch, dass er die Erweiterung seines kuratorischen Programms in einer krypto-neokolonialen Art der Globalisierung ohne Globalisierung suchte. Ausschließlich unter Beteiligung von Künstler_innen aus den gut situierten Milieus Londoner, New Yorker oder Berliner Großgalerien und weitgehend das installative Produkt einer prozessualen Praxis vorziehend, stellte Bourriaud eine privaten und institutionellen Sammler_innen schmeichelnde Ausstellung zusammen, die er überdies mit einem „Manifest“" versah, das seinem neuen Begriff oder Slogan der „Altermoderne“ Substanz verleihen sollte.

In Bourriauds Text ist viel von reisenden (bzw. jetsettenden) Künstler_innen und ihren Übersetzungsproblemen die Rede, von einer Welt, in der man eine „tiefreichende Evolution“ der Weltsicht und -wahrnehmung erlebe, von einer „neuen Moderne“, die nach ebenso neuen „Typen der Repräsentation“ verlange. ${ }^{5}$ Die Postmoderne, so Bourriaud, sei am Ende, Diskurse des Multikulturalismus und der Identität seien überholt und ersetzt worden durch eine „planetare Bewegung der Kreolisierung“, die allerdings weiterhin, darin wiederum echt postmodern, unterfüttert sei von „kulturellem Relativismus“ und „Dekonstruktivismus“, mit denen man sich erfolgreich gegen den Universalismus der Moderne und einen Traditionalismus der Rechten zur Wehr setzen könne. Die Kultur der nach-postmodernen „Altermoderne“ sei nicht länger ein vor allem westliches Phänomen, sondern erhebe sich zentrumslos und

4 Siehe z. B. Rancière, Jacques: „Probleme und Transformationen der kritischen Kunst", in: ders.: Das Unbehagen in der Ästhetik, Wien 2007 [zuerst Paris 2004]. Für einen neueren Überblick zu der Kontroverse um Bourriaud siehe: Miller, Jason: „Activism vs. Antagonism: Socially Engaged Art from Bourriaud to Bishop and Beyond", in: Field. A Journal of Socially-Engaged Art Criticism, Bd. 3, Winter 2016, S. 165-183.

5 Bourriaud, Nicolas: „Manifesto“ [Übersetzung d. Verf.], in: Website der Tate Gallery zur Ausstellung „Altermodern“, Tate Triennial, 3. Februar bis 26. April 2009, https://www.tate.org.uk/whats-on/tate-britain/exhibition/altermodern/ altermodern-explain-altermodern/altermodern-explained (20.08.2019). 
„polyglott“ aus „planetaren Verhandlungen“, aus „Diskussionen zwischen den Akteuren unterschiedlicher Kulturen“; sie kündige eine Verpflichtung auf abendländische Abstraktionen ebenso auf, wie sie sich von einer identitätspolitisch aufgeladenen Kultur der Postmoderne distanziere. „Unser Universum“, schreibt Bourriaud, „wird zum Territorium, dessen sämtliche Dimensionen in Zeit und Raum durchreist werden können." 6

Es geht mir nun nicht darum, die Leere und Vagheit solcher Kuratorenlyrik zu demaskieren, das wäre billig und wenig produktiv. Was hier allein interessieren soll, ist Bourriauds Verschiebung seines Relationalitätskonzepts aus dem Bereich des Begriffs einer Kunst, die ihren „theoretischen Horizont“ statt in einem „unabhängigen und privaten symbolischen Raum“ in „menschlichen Interaktionen“ und deren "sozialem Kontext“ findet, ${ }^{7}$ auf die von Mobilitäten und Kreolisierungen geprägte Bühne „planetarer“ Verhandlungen - wobei insbesondere Informationsverarbeitung, Übersetzungen und semiotische Wanderungen betont werden.

Bezeichnenderweise keine Rolle spielte im Zusammenhang der „Altermodern"-Ausstellung von 2009 und ihrer diskursiven Rahmung jenes Ensemble von Fragen, das knapp zehn Jahre später bei ähnlichen Gelegenheiten kunstinstitutioneller Globalisierungsrhetorik einfach nicht mehr ignoriert werden kann und von vorneherein ins Zentrum der Debatten um Gegenwartskunst gestellt wird: Keine kuratorische Leitung einer Ausstellung des Typs, den „Altermodern“ repräsentierte, würde Bourriauds Künstler_innen-Liste (die ausschließlich aus Einzelkünstler_innen besteht, keine Kollektive oder Organisationen enthält) unter dem Schlagwort einer ,anderen“ Moderne und einer „anderen“ Globalisierung präsentieren können, ohne scharfe Kritik an der überwiegend westlichen Perspektive auf das Planetarische und die geradezu unverhohlene Feier ökonomischer Privilegien erwarten zu müssen (und natürlich war dies auch 2009 im Grunde nicht mehr möglich). Ein Begriff wie Bourriauds „Kreolisierung“ gestikulierte zwar in Richtung einer Neuordnung der Einflusssphären und einer Dezentrierung dominanter Sprachformen, aber von den Intentionen und Argumenten eines Ansatzes der entschiedenen Dekolonialisierung der Kunstweltverhältnisse und ihrer Repräsentationsmodi, wie er allein angemessen gewesen wäre und inzwischen, folgerichtig und zwangsläufig, den internationalen Diskurs bestimmt, war er denkbar weit entfernt. Die Applikation postkolonialer Konzepte blieb letztlich ornamental. An einer Geschichte und Gegenwart der Konflikte, auf die eine dezidiert wissenspolitische Perspektive reagieren sollte, war Bourriaud nicht interessiert. Und doch markieren die Jahre um 2009 die Zeit, in der dekoloniale Konzepte und ihre Thematisierung globaler epistemischer Asymmetrien und Gewalt-

6 Ebd.

7 Bourriaud 2002, S. 5: „The possibility of a relational art (an art taking as its theoretical horizon the realm of human interactions and its social context, rather than the assertion of an independent and private symbolic space), points to a radical upheaval of the aesthetic, cultural and political goals introduced by modern art.“ 
verhältnisse nicht nur endgültig die Diskussionen innerhalb der Postcolonial Studies erreichten, sondern auch an den Orten der zeitgenössischen bildenden Kunst zunehmend Beachtung fanden. Der Reihe nach: Die Historikerin Ann Laura Stoler veröffentlicht 2009 mit Along the Archival Grain. Epistemic Anxieties and Colonial Common Sense eine wichtige Arbeit zu den „epistemic politics“ des Kolonialismus, die auf Vorarbeiten wie dem Aufsatz „Epistemic Politics: Ontologies of Colonial Common Sense" von 2008 basierte. ${ }^{8}$ Stolers Studien zu rassistischem Machtwissen in den Kolonien waren zwar nicht ohne Vorgänger. Die Art jedoch, wie hier epistemologische und ontologische Verfahren zur Herrschaftssicherung zum Untersuchungsgegenstand gemacht wurden, hatte um 2009 eine besondere Resonanz. Zwei der meistzitierten Autoren des neuen Dekolonialismus etwa veröffentlichten ebenfalls 2009 in der Zeitschrift Theory, Culture \& Society einflussreiche Artikel: Walter Mignolo, der argentinische Literaturwissenschaftler und Direktor des Center for Global Studies an der Duke University, betitelte seinen Beitrag „Epistemic Disobedience, Independent Thought and De-Colonial Freedom", womit er nicht- oder auch antiwestliche Epistemologie, Widerstand und Dekolonialität in einen Artikulationszusammenhang brachte; ${ }^{9}$ und der portugiesische Ökonomietheoretiker Boaventura de Sousa Santos fragte im Titel seines Artikels nach der Denkmöglichkeit eines „Non-Occidentalist West?“ und fügte in Anspielung auf Nicolaus Cusanus, Blaise Pascal und Lukian von Samosata den Untertitel „Learned Ignorance and Ecology of Knowledge“ an. ${ }^{10}$

De Sousa Santos ist weniger an neoheroischen Typen wie Bourriauds „homo viator" interessiert als an der cusanischen Figur des_der "gelehrten Unwissenden“, die sich seit dem Spätmittelalter und den theologischen Spekulationen über das Verhältnis des_der Einzelnen zur Unendlichkeit Gottes freilich gewandelt hat: Die_Der gelehrte Unwissende der Gegenwart ist sich einer epistemologischen Diversität bewusst, die potenziell unendlich ist und von jeder einzelnen Wissensform oder -weise nur sehr begrenzt erfasst werden kann. Das singuläre oder individuelle Nichtwissen im cusanischen Sinne ist durch ein unendlich plurales Nichtwissen ersetzt worden, das von einer unüberschaubaren Vielzahl der Wissensformen ausgehen muss. ${ }^{11}$ Der nichtwestliche Okzident

8 Stoler, Ann Laura: Along the Archival Grain. Epistemic Anxieties and Colonial Common Sense, Princeton, NJ 2009; dies., „Epistemic Politics: Ontologies of Colonial Common Sense“, in: The Philosophical Forum 39 (3), Herbst 2008, S. 349-361.

9 Mignolo, Walter D.: „Epistemic Disobedience, Independent Thought and De-Colonial Freedom“, in: Theory, Culture \& Society, Bd. 26, Nr. 7/8, 2009, S. 159-181.

10 Sousa Santos, Boaventura de: „A Non-Occidentalist West? Learned Ignorance and Ecology of Knowledge“, in: Theory, Culture \& Society, Bd. 26, Nr. 7/8, 2009, S. 103-125.

11 Ebd., S. 115: ,To be a learned ignorant in our time is to know that the epistemological diversity of the world is potentially infinite and that each way of knowing grasps it only in a very limited manner. In this respect, too, our condition is very different from Nicholas of Cusa's. Whereas the notknowing knowledge he postulates is singular and hence entails one learned ignorance alone, the learned ignorance appropriate to our time is infinitely plural, as plural as the possibility of different ways of knowing." 
der Vergangenheit und Gegenwart und die künftige Entwestlichung des Westens, die de Sousa Santos in Erinnerung bringt, in Aussicht stellt und einfordert, sind als das Resultat einer auf die westliche Epistemologie anzusetzenden Dekolonialisierung qua Pluralisierung zu verstehen, die auf verschüttete oder marginalisierte, das heißt auch: kolonisierte Denkweisen und Wissensformen im Inneren der westlichen Philosophie und Wissenssysteme aufbaut.

Mignolo sieht das alles ähnlich, nur legt er einen stärkeren Akzent auf Begriffe des Politischen im Zusammenhang mit Wissen. Seine Terminologie einer „politics of knowledge“ oder genauer: „geo- and body-politics of knowledge“, will über Donna Haraways Begriff der „situated knowledges“ hinausgehen. Dabei tut Mignolo ein bisschen so, als bleibe Haraways und andere standortfeministische Begriffsarbeit dort stehen, wo es eigentlich erst interessant werde, weil angeblich erst die Frage nach dem Wer, Wann und Warum der Konstruktion von Wissen gestellt werden müsse, um in Erfahrung zu bringen, weshalb die „eurozentrierte Epistemologie“ ihre "geohistorischen und bio-grafischen Verortungen“ so geschickt zugunsten der Behauptung „universalen Wissens“ und „universaler Wissenssubjekte“ kaschiert habe. ${ }^{12}$ Nur so, durch die Offenlegung der „Illusion einer Nullpunkt-Epistemologie“, könne jener „epistemische Ungehorsam“ entstehen, der zu einer effektiven Ent-Koppelung, zu Mignolos inzwischen berühmtem de-linking als dem zentralen Manöver der Dekolonialisierung führt, wie er sie als maßgeblich epistemologische Operation versteht. ${ }^{13}$

Denn „dekoloniales Denken“ setze eine ebensolche Ent- oder Abkoppelung, eine Ent-Beziehung (oder Ent-ziehung) von modernen, imperialen Wissensnetzen und der „kolonialen Macht-Matrix“ voraus, was zugleich eine Abwendung von der abstrakten Entität des Kapitalismus und eine Hinwendung zu „Menschenleben“ bedeute. ${ }^{14}$ Die Dekolonialisierung des Wissens geht nach Mignolo einher mit einer dekolonialen Herstellung, einem dekolonialen Machen von Wissen, einer Poiesis in Bezug auf Wissen - was übrigens auch die Anschlussfähigkeit seiner Argumentation an ästhetische Reflexion und Kunsttheorie gewährleistete. ${ }^{15}$

12 Mignolo 2009, S. 160.

13 Ebd.: „By setting the scenario in terms of geo- and body-politics I am starting and departing from already familiar notions of ,situated knowledges'. Sure, all knowledges are situated and every knowledge is constructed. But that is just the beginning. The question is: who, when, why is constructing knowledges? Why did eurocentered epistemology conceal its own geo-historical and biographical locations and succeed in creating the idea of universal knowledge as if the knowing subjects were also universal? This illusion is pervasive today in the social sciences, the humanities, the natural sciences and the professional schools. Epistemic disobedience means to delink from the illusion of the zero point epistemology."

14 Ebd., S. 178.

15 Ebd.: „Decolonial thinking presupposes de-linking (epistemically and politically) from the web of imperial knowledge (theo- and ego-politically grounded), from disciplinary management. A common topic of conversation today, after the financial crisis on Wall Street, is ,how to save capitalism'. A decolonial question 
Mignolo hatte damit um 2009 eine Reihe von griffigen Formulierungen, von catch terms im Angebot, die rasch die Runde machen sollten und nicht unmaßgeblich dazu beitrugen, dass in den Geistes- und Kulturwissenschaften, den humanities, wie im Kunstfeld eine konzeptuelle Neuorientierung stattfand. Die postkoloniale Kritik wurde gewissermaßen rekalibriert, von der Frage "Can the Subaltern Speak?" (Gayatri Spivak) in Richtung einer Vergegenwärtigung, eines Bewusst- und Präsentmachens der anhaltenden und durch den Neoliberalismus erneuerten Kolonialität und deren Gegnerin, der Dekolonialität, die gegen Erstere vor allem auf der Ebene des Wissens und Denkens vorgeht.

Im Jahr 2009 war es auch, dass der kamerunische Biotechnologe, Theoretiker und Kurator Bonaventure Soh Bejeng Ndikung in Berlin den Kunstraum SAVVY Contemporary: The Laboratory of Form-Ideas gründete, eine Plattform für Ausstellungen, Performances, Diskursveranstaltungen, Archivierungsarbeit und Residencies, die zunächst in der Richardstraße in Neukölln in einem Ladengeschäft, ab 2013 im ehemaligen Umspannwerk in Neukölln untergebracht war und seit 2016 im silent green Kulturquartier im Wedding angesiedelt ist. Bonaventure Ndikung verortete SAVVY Contemporary, das rasch zu einer Organisation mit zahlreichen Kurator_innen und Forscher_ innen angewachsen ist und sich inzwischen auf dem Weg in eine nicht mehr nur prekäre Konsolidierung zu befinden scheint, „an der Schwelle zwischen Begriffen und Konzepten von WESTEN und NICHT-WESTEN, vorrangig um diese $\mathrm{zu}$ verstehen und zwischen ihnen zu verhandeln, aber auch, um die Ideologien und Konnotationen zu dekonstruieren, die solchen Gebilden zugrunde liegen". ${ }^{16}$ „Dafür", so heißt es in einem jüngeren programmatischen Text, „scheint es angebracht, die kosmogenen Kräfte künstlerischen Schaffens zu beschwören, zusammenzurufen und anzuwenden, um uns von ihnen leiten zu lassen. Auf diesem Weg setzt SAVVY Contemporary auf die poetische Kraft künstlerischer Praxis, um Identitäten zu entnennen und umzubenennen, zu deinstituieren und wieder neu [zu] errichten, um auferlegte Stimmen verstummen zu lassen und unterdrückte Stimmen hörbar zu machen, in dem Bemühen, die Krise der Gefangenschaft zu lösen."17

would be: Why would you want to save capitalism and not save human beings? Why save an abstract entity and not the human lives that capitalism is constantly destroying?' In the same vein, geo- and body-politics of knowledge, decolonial thinking and the decolonial option place human lives and life in general first rather than making claims for the ,transformation of the disciplines'. [...] decolonial options, grounded in geo- and body-politics of knowledge, engage in both decolonizing knowledge and de-colonial knowledge-making, delinking from the web of imperial/modern knowledge and from the colonial matrix of power."

16 Ndikung, Bonaventure Soh Bejeng: „SAVVY Contemporary. The Laboratory of Form-Ideas. A Concept reloaded“, in: Website von SAVVY Contemporary, 2017, https://savvy-contemporary.com/site/assets/files/2811/savvy_concept_2017-1.pdf, hier zitiert aus der deutschen Übersetzung der Kurzfassung, https://savvy-contemporary.com/de/about/concept/\#1513199811-3371-1 (20.8.2019).

17 „Werde ein SAVVY friend“", in: Website von SAVVY Contemporary, 2018, https://savvy-contemporary.com/de/about/donate/ (20.8.2019). 
Aus dem Zitierten wird deutlich, wie weitgehend die Position, von der aus SAVVY Contemporary operiert, von post- und dekolonialen, aber auch feministischen und queeren Theoriebildungen geprägt ist, von der Annahme des Konstruktionscharakters jeder diskursiven und ideologischen Setzung und der daraus folgenden Möglichkeit dekonstruktiver Verschiebungen und Aufhebungen. Die Rolle der Kunst wird dabei in einer Arbeit an der Disidentifizierung und Deinstituierung gesucht, die zugleich eine Arbeit an Befreiung und Ausbruch sein soll. Formulierungen wie „kosmogene Kräfte künstlerischen Schaffens“ verweisen überdies auf eine vor- oder nichtmoderne, eine nichtwestliche, indigene Auffassung von Kunst als kosmischer, Welten generierender Potenz.

In einem weiteren Text (von 2017) spricht Ndikung in Anlehnung an de Sousa Santos über SAVVY Contemporary als einen „Raum für epistemologische Diversität“, in dem ein „anderes Wissen möglich“ sein solle. ${ }^{18}$ Haraway und ihr Begriff vom „god-trick“ westlicher Wissenschaft, dem ein „verkörpertes, kompliziertes, aktiv schauendes Auge“ entgegengehalten wird, führt zur Berufung auf „Politiken und Epistemologien der Verortung, der Positionierung und der Situierung“, in denen anstelle modern-westlicher „Universalität“ „Teilhabe“, „Befangenheit“ und „Parteilichkeit“ zur Bedingung des Gehörtwerdens und rationaler Wissensbehauptungen werden. Verkörperte, vom einzelnen und kollektiven Körper her eingenommene Perspektiven könnten vor diesem Hintergrund zu „associated situated knowledges“ führen, einem Wissen der Sozialökologie, d.h. der Beziehungen, der Relationalitäten, der Assoziationen und der Begleitungen, wobei Kunst und Ausstellungsmachen katalysierend wirken. ${ }^{19}$

18 Ndikung 2017, siehe auch, für eine leicht gekürzte und übersetzte Fassung dieses in verschiedenen Versionen zirkulierenden Textes: Ndikung, Bonaventure Soh Bejeng: „Anmerkungen zum epistemischen Ungehorsam. Oder: Verwirrung des Verstrickten und Beunruhigung des Besorgten - Fallstudie SAVVY Contemporary“, in: Busch, Kathrin et al. (Hg.): Wessen Wissen? Materialität und Situiertheit in den Künsten, Paderborn 2018 (Schriftenreihe des DFG-Graduiertenkollegs „Das Wissen der Künste“), S. 29-35, hier: S. 31.

19 Ndikung 2017, S. 2f.: „[...] SAVVY Contemporary is a space for epistemological diversity. A space that embodies and screams out Boaventura de Sousa Santos' postulation that ,Another Knowledge is Possible ‘ [...]. By moving away from what she calls ,god-trick', i.e. the all-seeing eye of Western science that considers itself the omniscient observer, Haraway offers the image of the embodied, complicated, actively seeing eye, which is a split and contradictory observer. Haraway's argumentations for politics and epistemologies of location, positioning, and situating, where partiality and not universality is the condition of being heard to make rational knowledge claims resonate in our practice. So we appropriate proposals on viewing from a body - always a complex, contradictory, structuring, and structured body, against the view from above, from nowhere, or from simplicity - and we push this further to what we will like to call, Associated Situated Knowledges' or just ,Associated Knowledges. ' While viewing from these bodies, one also puts them in relation, association and companion with each other and their sociopolitical ecology. In so doing, one not only puts the bodies, but also their embodied knowledges, histories, memories in association. In this respect, art and exhibition-making act as catalysts.“ 
Während Bourriauds Konzepte der „relationalen Kunst“ und „relationalen Ästhetik" vor etwa 20 Jahren vor allem eine Reaktion auf eine als steril und akademisch empfundene westliche Moderne und weder an weitergehende politische noch ontologische Forderungen geknüpft war, hat sich die Lage seither ganz offensichtlich grundlegend gewandelt. Schon im Jahr 2009 hätte diese Veränderung im Vergleich etwa zwischen Bourriauds Ausstellung "Altermodern" und der Gründung einer Institution wie SAVVY Contemporary erkannt werden können, aber natürlich gab es zu diesem Zeitpunkt auch schon viele andere Anzeichen für eine dezidiert politisch motivierte und ästhetisch argumentierende Infragestellung einer hegemonialen Ordnung des westlich dominierten Kapitalismus - einer Ordnung, die nicht zuletzt aufgrund der von Mignolo in seinem Text von 2009 erwähnten globalen Staatsschulden- und Finanzkrise und deren mit Steuergeldern bezahlten, aber letztlich nur scheinhaften ,Lösung' ihre letzten verbliebenen, ihrerseits überwiegend scheinhaften Legitimitätsreserven verbraucht hatte. Neue Beziehungen und Bezüglichkeiten stehen inzwischen auf der Agenda, was durch die Kollateralschäden neoliberaler Politik und Ökonomie zunehmend unumgänglich wird. Der Klimawandel betrifft nicht nur die Gegenwart und $\mathrm{Zu}$ kunft der Menschheit, sondern des gesamten Planeten und seiner menschlichen wie nichtmenschlichen Ökosysteme und Ressourcen. Zu den wichtigsten Begriffen der Debatte, etwa im Zusammenhang von Kämpfen um Klimagerechtigkeit, soziale Gleichstellung und die Rechte indigener Völker gehört eben gerade der Begriff der Beziehung, der Relation - der Beziehung zum Land, zur Erde, zu Menschen, Tieren, Pflanzen, Bodenschätzen, zu dem, was gewohnheitsmäßig im Westen immer noch ,Natur (im Unterschied zu ,Kulturc) genannt wird.

Indigene Intellektuelle wie die Maori Linda Tuhiwai Smith in ihrem Buch Decolonizing Methodologies. Research and Indigenous People von 1999 (einer zentralen Quelle etwa für Mignolos decoloniality-Epistemologie) oder die Dakota Kim TallBear in ihrer Forschung zu Indigenität, Genetik und Technoscience, aber auch zum Queering dessen, was sie „whitestream disciplinary thinking and ontologies" nennt, ${ }^{20}$ arbeiten die Epistemologien dieser Relationalität (oder Reziprozität) heraus. Im Vergleich etwa zu Bourriaud erweitern und vertiefen diese relationalen Epistemologien und reziproken Denkweisen jeden Begriff von ,relationaler Kunst'. Smith adressiert in ihrem - freilich nicht auf Kunst im engeren, westlich-modernen Sinne eingehenden - Buch die Notwendigkeit eines völlig neuen ethischen Habitus der Forschung. Sie spricht von „Wissensteilung“, von sharing knowledge, im Unterschied zum „Teilen von Information", weil die Verantwortung von Forscher_innen und Akademiker_innen eben darin bestehe, nicht nur ,pamphlet knowledge" zu teilen, sondern die „Theorien und Analysen“, mit deren Hilfe Wissen und Information konstruiert

20 TallBear, Kim: „An Indigenous Reflection on Working beyond the Human/ Not Human", in: GLQ, Bd. 21, Nr. 2/3 (Theorizing Queer Inhumanisms), 2015, S. 230-235, hier: S. 230. 
und repräsentiert werden. „The challenge always is to demystify, to decolonize",21 nur wenn dies geschehe, öffne sich Wissensproduktion auch Leuten, die über keine akademische Bildung verfügten. Denn theoretisch sei jedes Handeln Denken und Tun zugleich - und project workers, community activists oder Berater_innen seien, weil sie Daten erheben, Informationen sammeln, sichten, organisieren und zur Darstellung bringen, in einem nicht unmaßgeblichen Sinne als „Forscher_innen“ anzusprechen. Smiths ausdrückliche Absicht besteht daher auch darin, „to develop indigenous peoples as researchers“.22

Im Kontext des Graduiertenkollegs „Das Wissen der Künste“ ist es vielleicht notwendig, auf die wissenspolitische Differenz zwischen dem Projekt von Smith, die Anerkennung und Entwicklung indigener Leute als Forscher_innen zu befördern, und einer seit etwa zwei Jahrzehnten an den Kunsthochschulen geförderten Entwicklung von Künstler_innen zu Forscher_innen im Rahmen von artistic-research-Programmen hinzuweisen. Was auf den ersten Blick eine strukturelle Ähnlichkeit aufweist, erweist sich bei näherem Hinsehen als eine optische Täuschung - eine allerdings nicht ganz unschuldige Täuschung, denn die Adressierung von Künstler_innen als Angehörigen einer von magischen Ritualen und Meistererzählungen geprägten Stammesgesellschaft war lange Zeit nicht unüblich, weshalb die Bekehrung zu höheren wissenschaftlichen Qualifikationen als künstlerische Forscher_innen gern wie ein säkularer Ausweg oder gar Aufstieg dargestellt wurde, was freilich in vielen Fällen die Perspektive auf die Verwertungsdynamiken und Assessment-Realitäten einer akademischen Wissensökonomie versperrte. Die Anerkennung von Maori community workers als Forscher_innen mit eigenen, nur eben nicht auf der europäischen Aufklärung fußenden Methodologien argumentiert dagegen klar im Widerstand gegen die neoliberale Ökonomisierung des Wissens; sie erhebt einen Anspruch auf Forschung und research in einem sozialen Kampf um Anerkennung, der aber zugleich die Prämissen dieser Anerkennung in Zweifel zieht.

Auffällig an der Entwicklung seit etwa 2009/10 ist, in welchem Maße Fragen des Wissens, einer globalen Wissenspolitik und der Pluralität oder Diversität der Epistemologien, also der nicht länger im Sinne eines universellen abendländischen Rationalitäts- und Wissenschaftsverständnisses abzuhandelnden nichtwestlichen Wissensformen, auf die Selbstverständigung der Akteur_innen in weiten Teilen des Feldes der Gegenwartskunst eingewirkt haben und wie diese Fragen die Debatte seither sehr grundsätzlich formieren und prägen. Allein in Berlin lässt sich dies an vielen Stellen beobachten. Neben SAVVY Contemporary und den vielen Aktivitäten, in die die Plattform involviert ist, könnten (aus dem Blickwinkel des Jahres 2018) die zehnte Ausgabe der Berlin Biennale, die Konferenz „Knowledge Entanglements. Beyond Abyssal Thinking“ des DAAD-Künstlerprogramms (deren Titel einen Begriff von de

21 Smith, Linda Tuhiwai: Decolonizing Methodologies. Research and Indigenous People, London 2012, S. 17.

22 Ebd., S. 18. 
Sousa Santos aufnimmt) oder die im Wintersemester 2017/18 vom Graduiertenkolleg „Das Wissen der Künste“ veranstaltete Vortragsreihe „Künste dekolonisieren“ genannt werden. Dass die documenta 14 im Jahr 2017 ebenso wie manche der ihr vorausgegangenen Ausgaben und zahlreiche Biennalen der vergangenen Jahre ebenfalls unterschiedliche Aspekte und Facetten der Dekolonialisierung schwerpunktmäßig verhandelt haben und dass diese Verhandlungen in zunehmendem Maße auch solche über die Rolle der Kunst, der Künstler_innen und anderer im Kunstfeld produzierender Intellektuellen, Akademiker_innen, Kurator_innen, Aktivist_innen usw. sind, hängt nicht zuletzt damit zusammen, dass Wissen und Epistemologie zunehmend als Funktionen und Produktionsmodi der Kunst begriffen werden und die Frage nach der Gemachtheit des Wissens in den Blick rückt, die Mignolo in dem Begriff einer antinormativen poiesis fasst. Der Bezug auf Wissen und Epistemologie und deren Produktion suspendiert aber nicht die Frage nach deren Ökonomisierung, im Gegenteil. Eine neoliberale Wissensökonomie, in der die Epistemologie des Marktes zur fundamentalen Kategorie jeder Wissensproduktion wird, lässt weder die universitären noch die Kunst-Institutionen, noch deren außer- oder parainstitutionellen Supplemente und Widerlager unangetastet. ${ }^{23}$ Andererseits kann sich, gerade angesichts der Unausweichlichkeit wissenskapitalistischer Zugriffe, das Möglichkeitsfeld anderer, alternativer, postkapitalistischer und/oder am Konzept der Commons orientierten Ökonomien öffnen. Etwa im Bereich der Kämpfe um Landrechte und nachhaltiges Wirtschaften gehen Formen von aktivistischer Kollaboration, die sich der Verwertungslogik entziehen, und künstlerisch motivierte Praktiken vermehrt ineinander über.

Kunsthistoriker_innen wie T.J. Demos, die seit Jahren zum Zusammenhang von wissens- und forschungsbasierten, zumeist prozessual und langfristig angelegten Kunstprojekten und den Möglichkeiten eines dekolonialen, indigenen und ökologischen Aktivismus arbeiten, machen sehr deutlich, in welchem Maß Künstler_innen und ihre Kooperationen zum Widerstand gegen und zur Kritik an den Ursachen von Zerstörungen von Umwelten und Lebenswelten beitragen und dass künstlerische Praxis in diesen Zonen der Gegenwartskunst von epistemischer Experimentalität nicht zu trennen sind.

In Vorbereitung auf sein Buch Decolonizing Nature. Contemporary Art and the Politics of Ecology ko-kuratierte Demos im Jahr 2015 im Nottingham Contemporary die Ausstellung „Rights of Nature: Art and Ecology in the Americas", an der unter anderem Allora \& Calzadilla, Eduardo Abaroa, Ala Plástica, Marcos Avila Forero, Amy Balkin, Subhankar Banerjee, Mabe Bethônico, Ursula Biemann \& Paulo Tavares, Center for Land Use Interpretation, Minerva Cuevas, Jimmie Durham, The Otolith Group, Fernando Palma Rodríguez, Claire Pentecost und Abel Rodríguez teilnahmen. Wie Mignolo und andere bezieht Demos einen entscheidenden Teil seiner theoretischen

23 Dazu weiter im Detail: Holert, Tom: Knowledge Beside Itself. Contemporary Art's Epistemic Politics, Berlin 2020. 
Basis für die Arbeit mit den experimentellen Ökologien dieser gegen das Anthropozän, den Klimawandel und die Zerstörung von Lebensgrundlagen indigener Gemeinschaften gerichteten künstlerisch-aktivistischen Praktiken aus den Texten indigener Theoretikerinnen wie Smith, TallBear oder Zoe Todd. Und er folgert aus der Beschäftigung mit diesen Autorinnen, dass die Arbeit bei der Dekolonialisierung der eigenen Forschungsmethoden ansetzen und die Beziehung zu den „Geschichten der Kämpfe und Perspektiven der Kolonisierten, einschließlich indigener Kosmologien, subalterner Rechtsprechung und sozialer Bewegungen wo immer angebracht" suchen müsse auch in Reaktion auf die Kritik, die von indigener Seite an der Nichtanerkennung oder Ausklammerung von First-Nation-Intellektuellen aus den aktuellen Debatten um neue Materialismen, Vitalismen und Objekt-orientierte Ontologien geübt wird. ${ }^{24}$

Eine Konsequenz dieser Verbindungen von Umweltaktivismus, indigener Selbstbehauptung, nichtwestlichen Epistemologien, akademischer Forschung und visueller Praxis ist eine tendenzielle Auflösung oder ersatzlose Substituierung der Kategorie Kunst. Letztere taucht - als „contemporary art" - zwar noch im Untertitel von Demos' Buch auf, aber ein Netzwerk wie das global agierende Multimedia-Projekt World of Matter, das eine prominente Rolle in Demos' Narrativ einer auf die Dramatik der Lage reagierender dekolonialen, umweltaktivistischen und epistemologischen Kooperation spielt, kommt in seinem mission statement ganz ohne Bezugnahme auf Kunst aus. Zwar werden die Formate der Ausstellung, der öffentlichen Veranstaltung, der Publikation und der Online-Plattform genannt, aber es geht World of Matter ansonsten vor allem um die Produktion und Bereitstellung von ,visuellem Quellenmaterial“ als einem „Instrument für Bildungsprozesse, aktivistische Arbeit, Forschung und die Anhebung eines allgemeinen öffentlichen Bewusstseins" der Krise. ${ }^{25}$

Für Demos definiert World of Matter die Natur zum Schauplatz einer ästhetisch-konzeptuellen Spekulation um, mit der die sozialen Kämpfe gegen die Kontrolle durch Konzerne ernst genommen und die Entwicklungen des Natur-Rechte-Diskurses einbezogen würden. ${ }^{26}$ Im Vordergrund stehe der Beitrag zu einer „ökologischen Wissensallmende“, den ecological knowledge commons. Gefördert werden solle die freie Produktion von geteiltem Wissen, das offen, kollektiv und zugänglich bleibt - im Widerstand gegen staatlichprivatwirtschaftliche Überwachung und proprietäre Kontrollen des Internet. ${ }^{27}$ Eine dezidiert wissenspolitische Formierung der visuellen und forschenden Praxis wie bei World of Matter findet sich auch bei ähnlich gelagerten, wenn auch im inhaltlichen oder strategischen Detail sehr unterschiedlich ausge-

24 Demos, T. J.: Decolonizing Nature. Contemporary Art and the Politics of Ecology, Berlin 2016, S. 22-25.

25 „About the project. World of Matter“, in: Website von World of Matter, https://www.worldofmatter.net/about-project (29.08.2019).

26 Demos 2016, S. 28.

27 Ebd., S. 212. 
richteten Projekten wie Forensic Architecture, Center for Land Use Interpretation, Critical Art Ensemble, The Otolith Group, Casco oder Sarai. Hier ergänzen einander juristische Intervention, alternative Pädagogik, theoretisch-visuelle Spekulation, aktivistische Vernetzung und dekoloniale Perspektive in einem Maße, dass die wissenspolitische und kapitalismuskritische Dimension dieser Kollektive und Organisationen die Parameter des Ästhetischen und Künstlerischen in eine kritische Randlage zu drängen scheint.

Ein völliger Funktionsverlust des Ästhetischen ist dabei sicherlich nur bedingt zu diagnostizieren, zu sehr sind für die meisten oben genannten Gruppen Form-Fragen in Hinblick auf Rhetorik, Kommunikation und Begründung der institutionellen Verortung der eigenen Praxis von Bedeutung. Trotzdem verschieben sich die Gewichtungen, komponiert sich die Praxis in diesen Fällen anhand unterschiedlicher, selten von formalen und ästhetischen Prioritäten dominierter Anliegen. Eine entscheidende Rolle spielt die Dringlichkeit der planetaren Problemlage, aber ein tieferer Grund mag auch in der abjekten Verflechtung von contemporary art mit denselben Agenturen der Finanzialisierung zu finden sein, die auch aus Wäldern und Flüssen finanzialisierte assets machen. Was, wenn die intellektuellen Energien und Ambitionen, die noch vor nicht allzu langer Zeit in der Kunst eine Wirklichkeit verändernde Kraft gesucht und bisweilen gefunden haben, jetzt vermehrt in den Umbzw. Abbau eines Rationalitätstyps geleitet werden, der für den Schaden verantwortlich ist, den zu reparieren es vielleicht zu spät ist? Was, wenn Kunst in Wissenspolitik aufgeht? Wissenspolitik sich an die Stelle von Kunst setzt oder, weniger ultimativ formuliert, Kunst in Wissenspolitik ihre zeitgemäße Funktion erkennt?

Die Einklammerung des Kunstbegriffs ist nun keine neue Maßnahme, die Abschaffung der ,Kunst ${ }^{\star}$ ohnehin ein zentrales Anliegen der Avantgarden des 20. Jahrhunderts. Paradigmatisch an der aktuellen Situation mag daher vor allem sein, dass ,Kunst', einer vertrauten diskursiven Strategie des Postkolonialismus folgend, immer radikaler provinzialisiert wird. Durch die Gleichsetzung des Kunstbegriffs und der auf ihm gründenden Praktiken und Institutionen mit dem Projekt der westlichen Moderne, zu dem Siedlerkolonialismus, Sklaverei, Heteropatriarchat, Ausbeutung natürlicher Ressourcen und nicht zuletzt ein bestimmtes, andere Wissensformen ausgrenzendes Verständnis von Rationalität gezählt werden, erscheint ,Kunst' zunehmend als toxisch, wo ihre ontologischen Voraussetzungen in politische und ökonomische Verhältnisse rückübersetzt werden. Was künstlerische Autonomie angeblich zur Entsprechung aufklärerischer Zielbestimmungen demokratischer Vergesellschaftung macht, erweist sich in post- und dekolonialen Kritiken als Element der umfassenderen Bedrohung nichtmoderner sozialer und religiöser Praktiken und Epistemologien durch den Westen. Zugleich ist zu berücksichtigen, dass sich Begriffe von Kunst und vor allem ihren modernistischen Ausprägungen, wie z.B. Repräsentation und Abstraktion, im Zuge des Imperialismus global verbreitet und diskursiv abgelagert haben. 
Das heißt, eine von den Spuren der Moderne und ihren Kunstauffassungen gänzlich freie Ästhetik ist allenfalls im Rückgriff auf vor- oder außermoderne Praktiken des Bildes aufzufinden, nicht aber in den multiplen Modernen einer anders, mithin jenseits jeder Zentrum/Peripherie-Topologie zu verhandelnden Globalität. Ähnliches gilt für das Verhältnis zwischen westlichen und nichtwestlichen Rationalitäten und Wissensformen. Wie soll eine Befreiung vom Joch der westlich-modernen Rationalität aussehen, eine Befreiung, für die in diskursiven Formen und Formaten gekämpft wird, die entscheidend von ebendieser Rationalität geprägt sind, auch wenn sich diese zugleich Manövern des Angriffs, der Einklammerung, der Suspendierung ausgesetzt sieht? Triffit der Befund zu, dass die Legitimität von Kunst und die von Wissen zunehmend als in einem Wechselverhältnis stehend verhandelt werden, dass sich also künstlerische und epistemologische - und hier eben vor allem: wissenspolitische - Fragen zunehmend verschränken, dann folgt daraus eine Verkomplizierung der Möglichkeiten, überhaupt von Kunst (und vom Standpunkt ,der Kunst' aus) zu sprechen. Das Sprechen ist auch für diesen Text alles andere als unproblematisch. Darauf verweist schon der Umstand, dass ein Großteil der in ihm zitierten und diskutierten Texte von männlichen Autoren stammt. Selbst eine standortpolitische und damit eigentlich feministische Perspektive garantiert also nicht die erfolgreiche Abkoppelung von blinden Flecken und lenkenden Prädispositionen. Das betrifft auch die Schreibweise, die Rückversicherung über akademische Gepflogenheiten, die Routinen der Diskursprozessierung. Die daher notwendige Verkomplizierung allerdings ist kein zu lösendes, auf Finalität und Schließung hin zu steuerndes Problem, sondern eine Chance zur Proliferation, zur Verzweigung und zur Neuverknüpfung - auch und gerade für die Diskussion um situierte Wissen, epistemische Privilegien und Sprechpositionen im Kunstfeld.

\section{Amy Lien and Enzo Camacho The Angry Christ}

The Angry Christ sits behind the altar of a modernist church founded, like the Philippine nation that is its setting, in the aftermath of war. The figure's arms reach straight outwards, shaping his body into a large, graphic sign of unconditional love, or something else. Implanted in the very center of the scene is the Sacred Heart, wrapped in thorns, fringed in flames, and set atop Christ's red and blue robe, which appears less like clothing and more like a webbed expanse of vein, muscle, and sinew, not covering Christ's body but flaying it open, turning it inside out. This is supposed to be a depiction of the Last Judgement, but there is also something kinky happening here, some indulgent pleasure in the way iconography as codified communication can be 